\title{
Two-Dimensional Elemental Operator for Modeling the Vectorial Hysteresis of Soft Magnetic Composite Material
}

\author{
Wei Jie $\mathrm{Xu}^{1,2}, \mathrm{Na} \mathrm{Na}$ Duan ${ }^{1}$, Shu Hong Wang ${ }^{1}$ \\ ${ }^{1}$ State Key Laboratory of Electrical Insulation and Power \\ Equipment, School of Electrical Engineering \\ Xi'an Jiaotong University \\ Xi'an 710049, China \\ shwang@mail.xjtu.edu.cn
}

\author{
You Guang $\mathrm{Guo}^{2}$, Jian Guo Zhu ${ }^{2}$ \\ ${ }^{2}$ School of Electrical, Mechanical and Mechatronic Systems \\ University of Technology, Sydney \\ NSW 2007, Australia \\ jianguo.zhu@uts.edu.au
}

\begin{abstract}
This paper presents a two-dimensional elemental operator with biaxial anisotropy based on the physical mechanisms of the cubic textured magnetic materials, and deduces an analytical expression of the direct relationship between magnetic field strength $H$ and magnetization $M$ for a single elemental operator by the partial approximate substitutions. To verify the proposed model, the magnetic hysteresis of a soft magnetic composite material SOMALOY ${ }^{\mathrm{TM}}$ 500 under alternating excitations was simulated and compared with the experimental results obtained by a 3D magnetic property tester. The results suggest that the proposed approach can be a useful tool in the modeling of vectorial magnetic hysteresis and the calculation of iron loss in practical engineering electromagnetic field analysis.
\end{abstract}

Keywords-elemental operator; vectorial hysteresis model; biaxial anisotropy; soft magnetic composite material

\section{INTRODUCTION}

Model of vectorial magnetic hysteresis has been under investigation for many years and various approaches have been proposed [1]. A typical one is based on the superposition of an either infinite or finite set of Preisach operators with different orientations. However, the operator of the Preisach model is scalar in nature, and thus cannot model the vectorial phenomena faithfully. On the other hand, the Stoner-Wohlfarth (S-W) operator, which is considered to be a single-domain particle with uniaxial crystal anisotropy, is vectorial inherently [2]. This assumption, however, is inconsistent with the phenomena in cubic textured magnetic materials which have biaxial anisotropy in each crystal plane.

In this article, to overcome the shortcomings in previous models, we introduce a vector elemental operator with biaxial anisotropy based on the physical mechanism of the cubic textured magnetic materials, and deduce an analytical expression to directly describe the relationship of $H-M$ on the single elemental operator.

\section{MODEL DESCRIPTION}

In the proposed model, it is assumed that the magnetic material is composed of interacting elemental operator which possesses its own biaxial anisotropy and anisotropy constant.
Similar to the S-W model, the magnetization direction of each biaxial elemental operator is determined from the various energy contributions of the material anisotropy and applied field. Then, the energy of a biaxial elemental operator can be written as

$$
E=K \sin ^{2} \theta \cos ^{2} \theta-\mu_{0} H M_{S} \cos \left(\theta_{\mathrm{M}}-\theta\right)
$$

where $\theta_{\mathrm{H}}$ and $\theta$ are the angles of the applied field and the resultant magnetization, respectively, both with respect to the particle's anisotropic (easy) axis, $K$ is the anisotropy constant, and $M_{\mathrm{S}}$ the saturation magnetization. The first term represents the biaxial anisotropic energy, and the second the interaction energy associated with the external magnetic field and the particle magnetization.

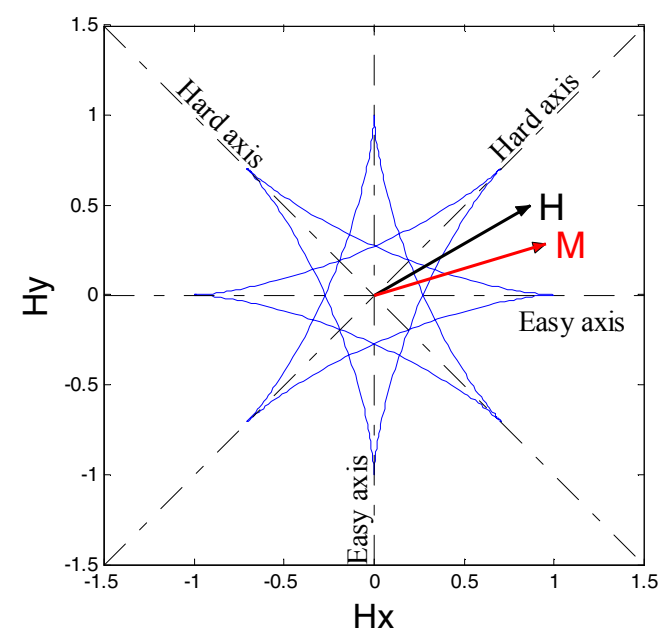

Figure 1. The biasteroid shape of the biaxial elemental operator with two easy axes and two hard axes.

Similar to the determination method of S-W particle, the stable orientation of the magnetization vector in the crystal structure can be obtained by minimizing the energy of the elemental operator.

Thus, the curve, which separates the regions with different energy extremes can be determined from

The authors gratefully acknowledge the support for this research from the China Scholarship Council (CSC) (201306280073). 


$$
\begin{aligned}
& \frac{\partial E}{\partial \theta}=\frac{1}{2} K \sin 4 \theta-\mu_{0} H M_{S} \sin \left(\theta_{H}-\theta\right)=0 \\
& \frac{\partial^{2} E}{\partial \theta^{2}}=2 K \cos 4 \theta+\mu_{0} H M_{S} \cos \left(\theta_{H}-\theta\right)=0
\end{aligned}
$$

The solution of the above equations related to $H_{\mathrm{x}}$ and $H_{\mathrm{y}}$, is the equation of a biasteroid

$$
\begin{gathered}
H_{x}=\frac{2 K}{\mu_{0} M_{S}} \cos ^{3} \theta\left(5-6 \cos ^{2} \theta\right) \\
H_{y}=\frac{2 K}{\mu_{0} M_{S}} \sin ^{3} \theta\left(5-6 \sin ^{2} \theta\right)
\end{gathered}
$$

where $H_{\mathrm{x}}$ and $H_{\mathrm{y}}$ are the components of the applied field $H$ along the two orthogonal easy axes.

As illustrated in Fig. 1, the biasteroid curve presents biaxial anisotropy with two orthogonal easy axes and two orthogonal hard axes. This property strictly agrees with physical mechanism of the cubic textured magnetic materials which have biaxial anisotropy in each crystal plane.

\section{IMPLEMENTATION}

With the new symmetry considerations on the magnetic anisotropy, an analytical way to describe uniaxial singledomain particles has been presented by Petrila and Stancu [3]. In the frame of partial approximate substitutions, to account for the magnetic interactions between elemental operators and the biaxial crystal anisotropy, a modified analytical expression can be proposed

$$
m(h, \theta)= \pm \frac{\sin \theta}{\sqrt{\frac{\left(h+h_{i}\right)^{2}}{h_{k}} \pm 2 \frac{\left(h+h_{i}\right)}{h_{k}} \cos \theta+1}}
$$

where $h_{k}=\frac{2 K}{\mu_{0} M}$ is the normalized anisotropy field, and $h_{i}$ the shifting field on the elemental operator. The plus sign is for the upper branch and the minus sign for the lower branch in a given hysteresis loop.

The bulk magnetization $M$ for a given applied field $H$ can then be calculated by the following integral

$$
M(H)=\iiint m(H, \theta) \mu\left(h_{i}, K\right) \cos \theta \mathrm{d} h_{i} \mathrm{~d} K \mathrm{~d} \theta
$$

where $\mu\left(h_{i}, K\right)$ is the distribution function of the elemental operator. In this model, the distribution can be described by a Gaussian-Gaussian distribution.

\section{EXPERIMENTAL VERIFICATION}

To verify the proposed model, the magnetic hysteresis of SMC material under alternating excitations is modeled and compared with the experimental results obtained by a 3D magnetic property tester [4]. The cubic SMC sample was cut from a preform of SOMALOY ${ }^{\mathrm{TM}} 500$.

Fig. 2 plots the simulated and measured hysteresis loops under two $50 \mathrm{~Hz}$ alternating magnetic field excitations of different magnitudes. As shown, the simulations agree well with the experiments.

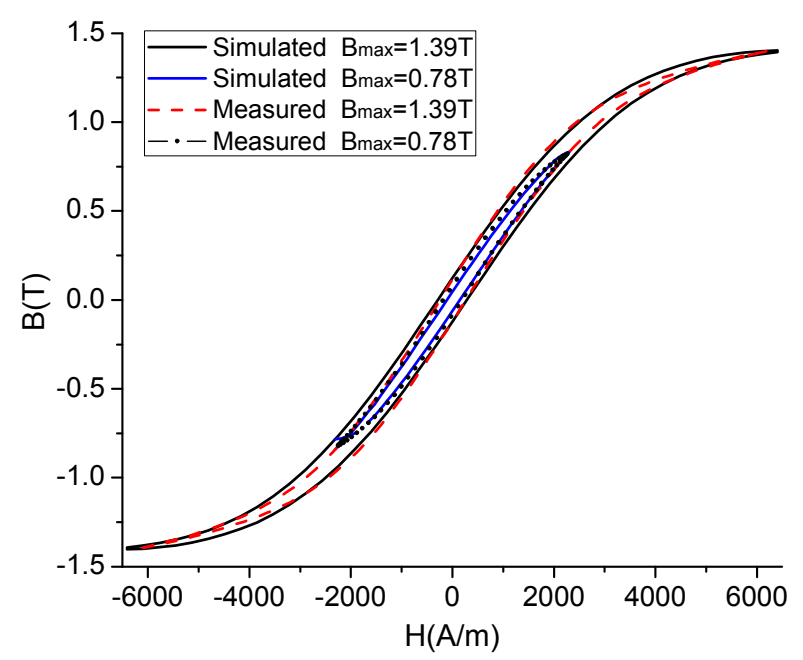

Figure 2. Comparison of measured and simulated B-H loops in a cubic SMC sample magnetized at $50 \mathrm{~Hz}$ with different alternating fields

\section{CONCLUSIONS}

A new approach for modeling of vectorial magnetic hysteresis based on a two-dimensional biaxial elemental operator has been presented including the principle, numerical implementation, and modeling. The simulations agree well with the experimental results of a cubic SMC sample measured by a $3 \mathrm{D}$ magnetic property tester.

\section{REFERENCES}

[1] I. D. Mayergoyz, "Mathematical models of hysteresis," New York: Springer-Verlag, 1991.

[2] E. C. Stoner, and E. P.Wohlfarth, "A mechanism of magnetic hysteresis in heterogeneous alloys," Philosophical Transactions of the Royal Society of London, vol. A240, no. 826, pp. 599-642, 1948.

[3] I. Petrila, I. Bodale, C. Rotarescu, and A. Stancu, "Linear and non-linear energy barriers in systems of interacting single-domain ferromagnetic particles," Physics Letters A, vol. 375, no. 39, pp. 3478-3482, 2011.

[4] Y. Li, Y. Liu, F. Liu, Q. Yang, and P. Ren, "Magnetic anisotropic properties measurement and analysis of the soft magnetic composite materials," IEEE Transactions on Applied Superconductivity, vol. 24, no. 5, pp. 1-4, 2014. 